Rend. Lincei Mat. Appl. 17 (2006), 87

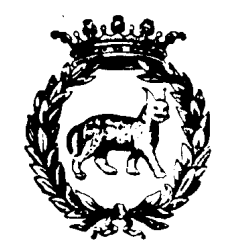

THIS ISSUE IS DEDICATED TO

\title{
GUIDO ZAPPA
}

ON THE OCCASION OF HIS $90^{\text {th }}$ BIRTHDAY

Born in Naples in 1915, member of the Accademia Nazionale dei Lincei since 1960, Guido Zappa is currently Emeritus Professor in the University of Florence. Although his main mathematical interests belong to group theory, and more specifically to abstract group theory-where he established relevant results on solvable groups, S-partitions, group varieties, and many others-one may well say that those interests and results stem from a unified and deep vision of mathematical research and science in general. That vision found a propitious atmosphere already in the graduate studies he attended to at the University of Rome and also in the personal contacts he had with Gaetano Scorza and Francesco Severi in those years, but it has a more remote origin in a lively interest in several aspects of science and culture and in their mutual relationship: interest and curiosity that, in his long and successful career, he was able to convey to many of those who had the opportunity and the privilege to work with him in the Universities of Naples and Florence, where he taught for several years.

The variety of the themes of the articles collected in this issue shows how far and how deeply this vision is shared by some of the colleagues and former students of Guido Zappa. 